\title{
Abwertung der Akupunkturleistung im EBM
}

Es sieht nicht gut aus für die Komplementärmedizin, sogar ganz schlecht für die Homöopathie. Wir müssen nun befürchten, dass auch die Akupunktur in den Abwärtssog mit hineingezogen wird, obwohl wir im Vorfeld Gespräche mit der Kassenärztlichen Bundesvereinigung (KBV) geführt haben und dort unsere Argumente vorbringen konnten.

Um 30 \% soll die Vergütung der Akupunktur im neuen einheitlichen Bewertungsmaßstab (EBM) 2020 sinken und in 5 min soll der Arzt dem nächsten Patienten zur Verfügung stehen. Berücksichtigt man, dass pro Akupunktur Materialkosten in Höhe von ca. $9 €$ entstehen und die - häufig alten - Patienten 20 min liegen, sich an- und ausziehen müssen, der Raum ist sicherlich insgesamt mindestens 45 min belegt, kann man davon ausgehen, dass fast niemand mehr in der Lage sein wird, sachgerecht und wirtschaftlich Akupunktur zu erbringen. Und in der Tat höre ich von den ersten Patienten, dass ihre Ärzte sie nur noch alle 2 Jahre akupunktieren könnten.

Sicher verfolgt die KBV mit dem Ziel, die sprechende Medizin zu stärken, ein wichtiges Ziel - allerdings steht insgesamt nicht mehr Geld zur Verfügung und somit geht es nur darum, das Vorhandene neu und anders zu verteilen. Schließlich kommt demnächst eine Flut von Liposuktionen auf uns zu und damit diese in den gedeckelten Topf passen, müssen eben für jede Liposuktion einige Hundert Patienten auf gute Akupunktur verzichten ...

Und das, obwohl sie sich in so vielen Studien als hochwirksam erwiesen hat! Akupunktur ist Zuwendung, BeHANDlung im besten Sinne, nah am Patienten, psychovegetativ entkoppelnd, stimmungsaufhellend, angstlösend.

Deutsche Zeitschrift für Akupunktur $2020 \cdot 63$ (1): 59

https://doi.org/10.1007/s42212-020-00239-7

Online publiziert: 20. Februar 2020

( ) Springer Medizin Verlag GmbH, ein Teil von

Springer Nature 2020
Wir hoffen, dass die KBV noch ein Einsehen hat und sich zumindest dem Argument, dass die Vorgaben des Gemeinsamen Bundesausschuss (G-BA), dass die Akupunktur, Arztzeit und Liegezeit zusammengerechnet, $30 \mathrm{~min}$ zu dauern hat, nicht verschließen wird. Für Ihre Unterstützung und Ihren Input bei allen Ihnen bekannten Entscheidern in der Kassenärztlichen Vereinigung $(\mathrm{KV})$ sind wir dankbar, damit uns die Akupunktur als wichtiges nichtmedikamentöses Schmerztherapieverfahren erhalten bleibt.

\section{Offener Brief zur Abwertung der Akupunkturleistung im EBM \\ Sehr geehrter Herr Kollege Gassen, sehr geehrter Vorstand der KBV,} nachdem unser Schreiben vom 18.12.2019 unbeantwortet blieb, wenden wir uns heute erneut an Sie.

Mit der Reform des EBM verfolgen Sie das wichtige Ziel, die sprechende und zuwendungsorientierte Medizin zu stärken.

Wir begrüßen diese Absicht, die auch von der Gesetzgebung gewünscht wird.

Leider wird die Akupunktur als zuwendungsorientierte und der sprechenden Medizin nahestehende Therapie durch die Reform des EBM deutlich abgewertet. Diese Abwertung widerspricht auch dem von der KBV in unserem gemeinsamen Gespräch 2013 geäußerten Standpunkt, dass Ärzte mit Akupunkturqualifikation auch in der Lage sein sollten, wirtschaftlich erfolgreich zu arbeiten.

Zusätzlich droht ein massiver Qualitätsverlust zulasten der Patienten.

Akupunktur kann mit dem neu zugeteilten Zeitvolumen von 5 min nicht mit angemessener Qualität durchgeführt werden.

Des Weiteren widerspricht die neue Zuteilung klar der Vorgabe des G-BA aus 2006, der besagt, dass die Akupunktur insgesamt $30 \mathrm{~min}$ dauern muss.

Somit ist festzustellen:

1. Wir wurden als Vertreter betroffener Ärzte nicht gehört.

2. Der Beschluss widerspricht den Vorgaben des G-BA und ist somit aus unserer Sicht unrechtmäßig.
3. Es droht ein massiver Qualitätsverlust. Diese wichtige und wirksame Kassenleistung ist von Ärzten nach Abwertung nicht mehr zu erbringen. Damit wird den Patienten eine evidenzbasierte Behandlungsform vorenthalten.

Diese Abwertung ist darüber hinaus unverständlich, zieht man Vergleiche für die Honorierung anderer Leistungen heran:

So wird die Akupunktur mit der Abwertung auf 160 Punkte und einem Zeitrahmen von 5 min schlechter gestellt als die Programmierung einer Medikamentenpumpe (Ziffer 30750, Erhöhung von 168 auf 180 Punkte), obwohl für Letztere weder ein Materialaufwand (Nadeln, Tupfer) noch ein zusätzlicher Raumbedarf NACH der Arztzeit (mindestens $20 \mathrm{~min}$ ) zu formulieren ist. Hier wird die Akupunktur ungleich schlechter gestellt aus Gründen, die für uns nicht nachvollziehbar sind.

Wir sehen die Akupunktur auch schlechter gestellt gegenüber der Standardtherapie als bisher, obwohl sie nach wissenschaftlicher Datenlage bei Rückenschmerzen und Gonarthrose mindestens gleichwertig ist bei deutlich besserem Risikoprofil.

Wir fordern Sie aus den genannten Gründen dringend auf die Abwertung der GOP 30791 zurückzunehmen und in einem gemeinsamen Gespräch zu einer tragbaren gemeinsamen Lösung zu finden.

\section{Mit freundlichen Grüßen \\ Naschmil Pollmann \\ Vorsitzende des Berufsverbandes}

Korrespondenzadresse

\section{Naschmil Pollmann}

Berufsverband Deutscher Akupunktur-Ärzte

Bernadottestr. 107, 22605 Hamburg,

Deutschland

naschmil@googlemail.com

Einhaltung ethischer Richtlinien

Interessenkonflikt. N. Pollmann gibt an, dass kein Interessenkonflikt besteht.

Für diesen Beitrag wurden von den Autoren keine Studien an Menschen oder Tieren durchgeführt. 